Physics Vol. 1, No. 4, pp. 229-247, 1965. Physics Publishing Co. Printed in Great Britain.

\title{
RENORMALIZATION EFFECTS FOR PARTIALLY CONSERVED CURRENTS*
}

\author{
S. FUBINI \\ Istituto de Fisica dell'Universitd-Torino \\ and CERN, Geneva \\ G. FURLAN** \\ CERN, Geneva \\ (Received 23 December 1954)
}

\begin{abstract}
In this paper it is shown how to get, through the use of suitable commutation relations, sum rules for the renormalization ratios between bare and dressed coupling constants. The method is completely general and it can be applied to any broken symmetry. As an illustration we have considered the cases of the weak vector current, both strangeness-conserving and strangeness-changing.
\end{abstract}

\section{Introduction}

ONE of the important facts in elementary particle physics is the existence of a "universality" property of the interactions. The oldest and most familiar example of such a situation is found in electromagnetism where the electromagnetic field is coupled in the same way to all the charged particles.

An analogous situation has been found in weak interactions and there are suggestions which propose to enlarge this concept also to the field of strong interactions.

In the context of quantum field theory the universality property gives rise to a delicate problem, if we want the statement to have a real meaning (also from the experimental point of view) and not a merely speculative one. In fact, it is necessary that the equality of the bare coupling constants originally inserted in the Hamiltonian can be reproduced in the equality of the renormalized quantities which have observable values. In the case of electrodynamics, the existence of the gauge group guarantees such a lack of renormalization due to other interactions. This provides the well-known concellation between propagators and vertex functions which is manifested by the Ward identity $Z_{1}=Z_{2}$.

- The research reported in this document has been sponsored in part by the Air Force office of Scientific Research AOR through the European Office, Aerospace Research, United States Air Force.

* Present address: Istituto di Fisica dell'Università-Trieste. 
The same problem arises in weak interaction physics: in this case it is necessary to introduce generalized gauge groups which make possible the same cancellations as in Q.E. D. Unfortunately the complete Lagrangian is only partially invariant with respect to these groups: in particular for the vector $\Delta S=0$ current the violation is introduced when we switch on the electromagnetic interactions, for the vector $\Delta S \neq 0$ current the violation is due to the socalled medium strong interactions which break $S U(3)$ symmetry. For the axial weak currents the situation is even more difficult since the presence of mass terms gives rise to a breaking of the invariance with respect to the gauge group.

Therefore an important and interesting problem is represented by the evaluation of the deviations of the renormalization ratio from unity which arise from the presence in the Hamiltonian of symmetry breaking terms. It is the aim of this paper to present a number of sum rules which will enable us, in principle, to obtain compact formulae for these corrections. In particular, the corrective terms can be expressed through observable quantities like physical matrix elements and coupling constants.

\section{General Formulation}

The main tool for deriving sum rules for the renormalization constants is the simultaneous use of the equal time commutation relations and of the matrix elements of canonical quantities between physical states. The equal time commutation relations involve interacting operators, and when the operators under consideration are currents or, more generally, the generators of a group, the validity of their commutation relations is preserved also if the group symmetry is broken. In addition, as pointed out for instance by Gell-Mann [1], the use of the explicit quantum field theoretical expressions for the currents allows us to introduce, in a natural way, a scale for the matrix elements of the same quantities. It is useful, in order to understand the underlying idea of the method, to recall the way the renormalization constants come into the game and the procedure for deriving sum rules for them.

a) As a starting point we consider a neutral, scalar interacting field $\phi(x)$.

The general properties of any theory, independent of the form of the coupling, are given by the usual invariance and spectral requirements and by the canonical commutation relations

$$
[\phi(\vec{x}, t), \dot{\phi}(\vec{y}, t)]_{-}=i \delta(\vec{x}-\vec{y})
$$

where the fields are the unrenormalized ones. The renormalization constant comes in when we consider matrix elements of the $\varphi$ field between physical states. Thus we define the constant $Z_{3}$ through the relation

$$
\left.\left\langle 0|\Phi(x)|_{\varphi}\right\rangle=\sqrt{z_{3}}<0\left|\varphi_{0}(x)\right|_{\varphi}\right\rangle
$$

where $\phi_{0}$ is the free field, and $|\cap\rangle$ and $|\phi\rangle$ are the true vacuum and one-particle states, respectively. It is clear that we can choose another normalization for the matrix element (2.2) introducing a renormalized field $\varphi_{R}(x)=Z_{3}^{-1 / 2} \varphi(x)$ such that

$$
\left.<0\left|\varphi_{R}(x)\right|_{\phi}>=<0\left|\varphi_{0}(x)\right|_{\varphi}\right\rangle
$$

On the other hand

$$
\left[\varphi_{R}(\vec{x}, t), \dot{\varphi}_{R}(\vec{y}, t)\right]_{-}=i z_{3}^{-1} \delta(\vec{x}-\vec{y})
$$


In other words, one can normalize to the free field form either the commutation relations or. the one particle matrix elements but, if we consider both, we have to deal with the renormalization constant $Z_{3}$ which summarizes the effect of the interaction at large distances or small energies $(<m)$.

Moreover using the well-known spectral representations of Källen-Lehmann [2] for the Green functions, it is possible to deduce the sum rule

$$
Z_{3}{ }^{-1}=1+\int_{M_{0}^{2}}^{\infty} \sigma\left(a^{2}\right) d a^{2}
$$

The weight function $\sigma\left(a^{2}\right)$ which contains the dynamical aspects of the theory is given by a sum of physical matrix elements of the form $<0||_{R} \mid n$ part $>$. Obviously in the free case these latter vanish and $Z_{3} \rightarrow 1$.

b) Analogous considerations and sum rules have been given in the framework of the static model by M. Cini and one of us [3]. The line of reasoning is the following: one starts from a non-renormalized Hamiltonian which contains the bare coupling constant $f_{0}$ and one considers next its matrix elements between physical states. The explicit expressions for these expectation values contain now a new coupling constant $f$, the dressed or renormalized one, and the link between the two is $f_{0}=Z f$.

The thing can be also expressed in an equivalent way as follows. If we limit ourselves, for simplicity, to the isotopic spin variables the expectation value of $f_{0} \times 7$ between physical one-nucleon states is, on general invariance grounds, *

$$
\left.<\psi_{s}\left|f_{0} \tau^{\alpha}\right| \psi_{s} \cdot\right\rangle=f_{\tau_{s}}^{\alpha}{ }^{\circ}
$$

If we want to introduce "renormalized" $\tau^{\alpha}$ operators, such that their matrix elements between dressed states are the same as between bare nucleon states, we can define $\tau_{R}^{\alpha}=Z_{\pi^{\alpha}}^{\alpha}$. But again the commutation relations involve the quantity $Z$

$$
\left[\tau_{R}^{\alpha}, \tau_{R}^{\beta}\right]_{-}=i \varepsilon_{\alpha \beta \gamma^{\top}}{ }^{\gamma} \times Z
$$

From these and similar relations it is easy to derive closed expressions for $Z$ and $f^{2}$. For instance, from

$$
\left[\tau_{R}^{(+)}, \tau_{R}^{(-)}\right]_{-}=Z_{\tau_{R}}^{(3)}
$$

we get, taking the expectation value between proton states

$$
Z=1+\left(\left\langle P\left|\left[\tau_{R_{1}}^{(+)} \tau_{R}^{(-)}\right]\right| P\right\rangle-\left\langle P\left|\tau_{R}^{(+)}\right| N\right\rangle\left\langle N\left|\tau_{R}^{(-)}\right| P\right\rangle\right)
$$

and the matrix elements on the r.h.s. can be immediately related to the pion-nucleon scattering amplitude. Once more we remark that the deviation of $Z$ from one is related to the presence

- As is well known this displays the fact that, owing to the interaction with the plons, $\vec{T}$ alone is not a good quantum number and the physical states are elgenstates of $\vec{T}=\vec{t}+\vec{T} / 2$, which is the conserved quantity. 
of nondiagonal terms which are switched on with the interaction.*

c) To conclude we want to discuss in some detail the role of the nondiagonal terms in the problem of the absence (or presence) of renormalization for a conserved (or partially conserved) current. To deal with a celebrated example, let us consider the case of a strongly interacting "proton" whose interaction with an external e.m. field is described by the Lagrangian density

$$
L_{\text {e... }}=e_{0} \bar{\psi} \gamma_{\mu} \psi A_{\mu}^{\text {ext }}=Z_{1} e \bar{\psi}_{R} \gamma_{\mu} \psi_{R} A_{\mu}^{\text {ext }}
$$

The $\psi, \psi_{R}$ are the unrenormalized and renormalized fields $\left(\psi_{R}=Z_{2}^{-1 / 2} \psi\right)$. For simplicity we consider the e.m. interactions to the first order so that $Z_{2}, Z_{1}$, the wave function and vertex renormalization constants, are due to the effect of the strong interactions only. Moreover " $e^{n}$ is the renormalized charge $e=Z_{2} Z_{1}^{-1} e_{0}$. We want to show that, as a consequence of charge conservation, a sum rule for $Z_{1} / Z_{2}$ necessarily gives $Z_{1} / Z_{2}=1$ and we will recognize the mechanism which produces this result [5]. To this end we introduce the total charge

$$
Q=\int e_{0} \bar{\psi} \gamma_{0} \psi d \vec{x}
$$

and we remember that the operative definition of the charge is

$$
e=\langle P|Q| P\rangle
$$

Using the equal time commutation relations (at $t=0$ ) we have that

$$
[Q, \psi]_{-}=-e_{0} \psi
$$

now

$$
\begin{gathered}
<0\left|[Q, \psi]_{-}\right| P>=\Sigma_{\alpha}<0|Q|_{\alpha}>\alpha|\psi| P>- \\
-\Sigma_{B}<0|\psi|_{\beta}><\beta|Q|_{P>}
\end{gathered}
$$

with $\mid P>$ being the physical proton state. Owing to the fact that $[Q, H]=0$, the charge is diagonal and there are no allowed transitions from the vacuum to any other state (the definition $<0|Q| 0>=0$ is understood). With the same argument in the second term we are left only with the state $\mid \beta>$ which contains one proton (with the same momentum as $P$ ). Thus

$$
<0\left|[\Omega, \psi]_{-}\right| P>=-\langle 0|\psi| P>P|Q| P>=-e<0|\psi| P\rangle
$$

and by comparison with (2.11) we get $e_{0}=e$ or $Z_{1} / Z_{2}=1$, the well-known Ward identity.

This reasoning can of course be generalized to all kinds of particles and conserved "charges". If a conserved charge $Q$ has the commutation rule with a field $\psi_{A}$

- Analogous considerations can be developed in the fully relativistic case and the use of the computation relations between the total components of isotopic spin becomes a source of information for electromagnetic form factors [4]. 


$$
\left[?, \psi_{A}\right]_{-}=-\alpha_{A} \Psi_{A}
$$

i.e., the field $\psi_{A}$ transforms like $\psi_{A} \rightarrow e^{-i \alpha_{A}} \psi_{A}$ under the gauge group generated by $Q$ we get

$$
<A|\cap| A>=\alpha_{A}
$$

which corresponds to a generalized Ward identity. Under strong and electromagnetic interactions, three such charges are conserved: the electric charge?, the baryonic charge $P$ and the hypercharge $Y$, which give rise to three independent identities of the kind (2.15), For example we shall get

$$
\begin{array}{ll}
<P|Q| P>=e & <P|Y| P>=1 \\
<\pi^{+}|Q| \pi^{+}>=e & <\pi^{+}|Y| \pi^{+}>=0
\end{array}
$$

which will be used in the following sections. Naturally the problem now is to generalize the previous considerations to other "charges", appearing for example in the weak interaction theory and which are only partially conserved, i.e., which commute only with a part of the total Hamiltonian. In those cases which will be discussed in the next sections, we expect nonvanishing contributions from non-diagonal matrix elements.

Summarizing the arguments of this section, the method which is being proposed is based on the following points:

1) Sum rules of the Källen-Lehmann type relate the renormalization corrections to the offdiagonal matrix elements of Heisenberg operators.

2) In the case of partial (or total) invariance under gauge transformations, operators like the charge are nearly constants of the motion and their non-diagonal matrix elements are small, of the same order as the symmetry violating interaction.

In the examples discussed in this section (a) and (b) only make use of point (1), on the other hand example (c) as well as the cases to be discussed in the following sections make essential use of the combination of points (1) and (2).

In the next sections we will illustrate these considerations by studying the following cases of physical interest: the renormalization of the weak vector current $\Delta S=0$ due to electromagnetic interactions (Section 3 ) and the renormalization of the weak vector current $\Delta S=\Delta$ ? due to the part of the strong Hamiltonian which breaks $S U(3)$ symetry (Section 4 ). In addition we will discuss also the possibility of more general sum rules and their possible applications (Section 5).

\section{Vector Strangeness Conserving Current}

The near equality of the coupling constant for the vector part of the nuclear $\beta$ decay with the coupling constant measured in $\mu$ decay led Feynman and Gell-Mann [ 6 ] to the hypothesis of the non-renormalization of the weak vector current. We start from the assumption of universality of the weak interactions, which states the equality of the bare coupling constants. As a consequence of the isotopic invariance of strong interactions, the weak vector current $\Delta S=0$ is conserved and this fact guarantees the exact universality, i.e., the equality of the observed coupling constants of $\beta$ and $\mu$ decay apart from electromagnetic effects. In other words, 
in the same manner as in the electromagnetic example discussed in the last section, we are sure that renormalization effects due to strong interactions do not change the $\beta$ decay coupling constant. Obviously these conclusions hold as long as we neglect the electromagnetic interactions which break isotopic symmetry. In this section we mean to evaluate the deviation of $G_{\beta}$ from its unrenormalized value $G_{0}$, i.e., we want to derive a sum rule for the quantity $R_{\beta}=G_{0} / G_{N P}$

Let us start by defining the weak vector currents

$$
\begin{aligned}
& J_{\mu}^{( \pm)}=G_{0}\left[I_{\mu}^{(1)} \pm i I_{\mu}^{(2)}\right]_{-}=G_{0} I_{\mu}^{( \pm)}=G_{0}\left(\bar{\psi}_{n} \gamma_{\mu}{ }^{\top}{ }^{(1} \psi_{n}+\ldots\right)= \\
& =G_{N P} Z_{1}(N P) \bar{\psi}_{n}{ }^{R} \gamma_{\mu}{ }^{\top} \Psi_{n}{ }^{R}+\ldots
\end{aligned}
$$

which, with $J_{\mu}^{(0)}$, form an isotopic triplet.* In the second formula the nucleonic fields and coupling constant are the renormalized ones. There can be renormalization effects due to electromagnetic interactions (that we will consider in first approximation on $l y$ ) and to strong interactions (which will be taken into account exactly). We have $G_{N P}=7_{1}^{-1}(N P) Z_{2}^{1 / 2}(P) Z_{2}^{1 / 2}(N) G_{0}$ and we remark that, owing to electromagnetic effects, the wave function renormalization constants of proton, $Z_{2}(P)$, and neutron, $Z_{2}(N)$, are different. In addition there will be different renormalized coupling constants $\left(G_{N P}, G_{K K}, G_{\pi \pi} \ldots\right)$ and renormalization constants $Z_{1}(N P)$, $Z_{1}\left(K K^{\prime}\right), Z_{1}(\pi \pi) \ldots$ for each part of the current.

If we introduce the "weak chargen

$$
\Upsilon^{( \pm)}=\int J_{0}^{( \pm)} d \vec{x}=G_{0} I_{ \pm}
$$

an operative definition of $G_{N P}$ is given by the relations

$$
G_{N P} \delta\left(\vec{p}_{2}-\vec{p}_{1}\right)=\left\langle P\left(p_{2}\right)\left|Q^{(+)}\right| N\left(p_{1}\right)\right\rangle=\left\langle N\left(p_{2}\right)\left|Q^{(-)}\right| P\left(p_{1}\right)\right\rangle
$$

with $|P>| N>$, physical proton, neutron states. Analogously

$$
G_{\pi \pi} \delta\left(\vec{p}_{2}-\vec{p}_{1}\right)=\left\langle\pi^{+}\left(p_{2}\right)\left|Q^{(+)}\right| \pi^{0}\left(p_{1}\right)\right\rangle=\left\langle\pi^{0}\left(p_{2}\right)\left|Q^{(+)}\right|_{\pi^{-}\left(p_{1}\right)}\right\rangle
$$

and so on.

Our discussion is based on the use of the commutation relations

$$
\left[?^{(+)}, Q^{(-)}\right]_{-}=G_{0}{ }^{2}\left[I_{+}, I_{-}\right]_{-}=G_{0}{ }^{2} 2 I_{3}=2 G_{0}{ }^{2}\left(Q-\frac{Y}{2}\right)
$$

where? is the total electric charge in units of $e$ and $Y$ is the hypercharge.

If we want now to get from equation (3.5) a sum rule for a particular coupling constant, it is sufficient to take the expectation value between convenient physical states. In particular - Actually we have written explicitly for $J_{\mu}^{( \pm)}$only the nucleonic terms but the contribution
due to other fields is implicit. 
we consider the matrix element of (3.5) between physical proton states. We get

$$
<P\left(p_{2}\right)\left|\left[Q^{(+)}, Q^{(-)}\right]-\right| P\left(p_{1}\right)>=G_{0}^{2} \delta\left(\vec{p}_{2}-\vec{p}_{1}\right)
$$

Then we introduce in the $1 . \mathrm{h} . \mathrm{s}$. of equation (3.6) a complete set of intermediate states and we select the contribution from the physical neutron state:

$$
\begin{aligned}
G_{0}{ }^{2} \delta\left(\vec{p}_{2}-\vec{p}_{1}\right)= & G^{2}{ }_{N P} \delta\left(\vec{p}_{2}-\vec{p}_{1}\right)+\Sigma_{\alpha \neq N}<P\left|Q^{(+)}\right| \alpha><\alpha\left|Q^{(-)}\right| P>- \\
& -\Sigma_{\beta}<P\left|Q^{(-)}\right|_{\beta>\beta}>\left.Q^{(+)}\right|_{P>}
\end{aligned}
$$

Let us now make explicit use of the fact that the strong interaction Hamiltonian commutes with $Q^{( \pm)}$so that the variation in time of $Q^{( \pm)}$is due only to the electromagnetic part.

$$
i \dot{Q}^{( \pm)}(t)=\left[Q^{( \pm)}, H\right]_{-}=\left[Q^{( \pm)}, H_{\bullet .}\right]
$$

We can use the explicit expression for $H_{\text {e. }}$

$$
H_{e .}=e \int J_{\mu}^{e m} A_{\mu} d \vec{x}
$$

to get

$$
\begin{gathered}
i \dot{Q}^{( \pm)}=\left[Q^{( \pm)}, H_{e \pm}\right]=\mp e \int J_{\mu}^{( \pm)} A_{\mu} d \vec{x} \\
=\mp e G_{0} \int J_{\mu}^{( \pm)} A_{\mu} d \vec{x}=+e G_{0} H^{( \pm)}
\end{gathered}
$$

For the corresponding matrix element it follows integration that

$$
\left.<a\left|Q^{( \pm)}\right| b\right\rangle= \pm . e G_{0} \frac{\left.<a\left|H^{( \pm)}\right| b\right\rangle}{E_{a}-E_{b}-i \varepsilon}
$$

Where the - iE prescription has been introduced to specify the free behaviour at $t=-\infty_{0}$ *

Finally we can write for equation (3.7):

where

$$
\frac{G_{0}^{2}}{G_{N P}^{2}}=\frac{Z_{1}^{2}(N P)}{Z_{2}(N) Z_{2}(P)}=1+e^{2}(A-B)
$$

- As can be seen by looking at equation (3.12), it would be the same to specify the $t=+\infty$ behaviour through a + ie prescription. 


$$
A \delta\left(\overrightarrow{\vec{p}}_{2}-\vec{p}_{1}\right)=\frac{G_{0}^{2}}{G_{N P}^{2}} \times \sum_{\alpha \neq N} \frac{<P\left|H^{(+)}\right| \alpha><\left|H^{(-)}\right| P>}{\left(E_{P}-E_{\alpha}-i \varepsilon\right)\left(E_{P}-E_{\alpha}+i \varepsilon\right)}
$$

and there is an analogous relation for B. Equations (3.12) and (3.13) show two important features. First of all, the corrections are of order " $e^{2 n}$ owing to the fact that there are two (smal1) non-diagonal matrix elements of $Q^{( \pm)}$. "Secondly the presence of the squared denominators gives a strong damping factor which ensures a certain control on the higher mass states.

Up to now our treatment is completely general in the sense that there are no approximations. If we want to limit ourselves to the $e^{2 n}$ order, we assume that the operators which enter in $H^{( \pm)}$are varying only according to the strong interactions and we can consider the currents $J_{\mu}{ }^{(t)}$ as conserved. In the same way, in the sum on the r.h.s. we can consistently take $G_{0}^{2} \simeq G_{N P}^{2}$ so that this dependence disappears.

Then, in the same order, we retain in the sum (3.13) the contribution from states $\mid \alpha>$, $\mid \beta>$ containing a single photon, other states leading to $e^{4}$ terms. Extracting explicitly the photon contribution, and summing over momenta and polarizations, we find that $A$, for instance, can be put in the form

$$
A=\int \frac{d \vec{q} d q_{0}}{2 \omega_{q} E_{P}} g_{\mu \nu} W_{\mu \nu}^{(a)} \frac{1}{\left(q_{0}-\omega_{q}\right)^{2}+\varepsilon^{2}}, \quad \omega_{q}=|\vec{q}|
$$

where we have defined

$$
W_{\mu \nu}^{(a)}=(2 \pi)^{3} E_{P} \bar{\Sigma}_{P} \Sigma_{a}<P\left|I_{u}^{(+)}\right| a><\left|I_{v}^{(-)}\right| P>\delta(q+p-P)
$$

and $p$ is the total momentum of the state $\mid a>, p^{2}=M^{2}$, and $P$ is the momentum of the proton.

Taking into account $J_{\mu}{ }^{( \pm)}$conservation (which requires $q_{\mu} W_{\mu \nu}^{(a)}=W_{\mu \nu}^{(a)} q_{\nu}=0$ ), $W_{\mu \nu}^{(a)}$ can be put into the standard form (see, for instance, Ref. [7])

$$
\begin{gathered}
W_{\mu \nu}^{(a)}=\left(g_{\mu \nu}-\frac{q_{\mu} q_{\nu}}{q^{2}}\right) W_{1}^{(a)}\left(q^{2}, q \times P\right)+ \\
+{ }_{m_{n}}{ }^{2}\left(P_{\nu}-q_{\nu} \frac{P \times q}{q^{2}}\right)\left(P_{\mu}-q_{\mu} \frac{P \times q}{q^{2}}\right) W_{2}(a)\left(q^{2}, q \times P\right)
\end{gathered}
$$

- Equation (3.11) has been explicitly used to evaluate the "off-diagonal" elements $\left\langle P\left|Q^{( \pm)}\right| \alpha\right\rangle$. It is anusing to consider the analogous relation

$$
\left.<P\left|Q^{(+)}\right| N\right\rangle=e G_{0} \frac{\left\langle P\left|H^{(+)}\right| N\right\rangle}{m_{P}-m_{N}}
$$

One rerifies imediately that this matrix element is indeed of order zero by noticing that

$$
\left\langle P\left|H^{(+)}\right| N>\simeq \int<P\left|I_{\mu}{ }^{( \pm)} A_{\mu}\right| N>\right.
$$

is of order e and $m_{P}-m_{N}$ is of order $e^{2}$. 
so that finally

$$
A=\int \frac{d \vec{q} d q_{0}}{2 \omega_{q} E_{P}\left[\left(q_{0}-\omega_{q}\right)^{2}+\varepsilon^{2}\right]}\left\{3 W_{1}^{(a)}+\frac{W_{2}^{(a)}}{m^{2} n}\left(P^{2}-\frac{(P \times q)^{2}}{q^{2}}\right)\right\}
$$

Equation (3.17) and an analogous formula for $B$ give the electromagnetic corrections to the renormalization ratio in terms of observable quantities. In fact the form factors $W_{1,2}$ describe the vector contribution to the elastic and inelastic neutrino-nucleon scattering. Moreover using the non-renormalization hypothesis they can be related to the elastic and inelastic electron-nucleon scattering amplitudes. * An explicit evaluation of these terms is now in progress together with a discussion of the other radiative corrections responsible for the difference between the observed values of $G_{\beta}^{2}$ and $G_{\mu}^{2}$.

\section{Strangeness Changing Current}

Let us turn to the case of the vector part of the strangeness-changing current, $\Delta S=\Delta Q$. It is well known that the use of the $S U(3)$ group [9] has led to a rather successful classification of the elementary particles and their interactions. In particular it is assumed that the weak vector current, like the electromagnetic current, is a component of the unitary spin current. Thus the vector currents form an unitary octet which is conserved when the symmetry is not broken and this result generalizes the conserved vector current hypothesis, which takes the $\Delta S=0$ current as the isotopic spin current. For simplicity we shall concentrate on the case of $K, \pi$ transitions; the generalization to other particles will not be discussed here.

The form of the current is

$$
\begin{gathered}
S_{\mu}^{\nu(+)}=G_{0 s}\left[I_{\mu}^{(u)}+i I_{\mu}^{(s)}\right]=G_{0 s} K_{\mu}^{(+)}=i G_{0 s}\left\{\phi\left(k^{+}\right) \partial_{\mu} \varphi\left(\pi^{0}\right)-\right. \\
\left.-\partial_{\mu} \varphi\left(k^{+}\right) \times \varphi\left(\pi^{0}\right)+\sqrt{2}\left[\varphi\left(\pi^{+}\right) \partial_{\mu} \varphi\left(k^{0}\right)-\partial_{\mu} \varphi\left(\pi^{+}\right) \times \varphi\left(k^{0}\right)\right]\right\}+\ldots
\end{gathered}
$$

The field operators are the unrenormalized ones and $G_{0 s}$ is the bare coupling constant, which according to a recent proposal [10] is in a fixed ratio, given by $\tan \theta$, to $G_{0}$ the bare coupling constant of $\beta$ decay.

In the limit when $S U(3)$ symmetry holds, $S_{\mu}^{\nu( \pm)}$ is conserved so that $G_{0 s}$ is not renormalized in the sense that it is not affected by the "very strong" interactions. Before discussing the renormalization effects, due to "medium strong" interactions, we would like to specify the definition of the renormalized coupling constant and the number of sum rules necessary to get information about all the quantities of interest.

Let us start by considering the matrix element of $S_{\mu}^{\nu(+)}$ between physical $\pi, K$ states. On invariance grounds we can write

- Analogous sum rules for the electromagnetic mass difference between proton and neutron have been derived by Cini, Ferrari, Gatto and Cottingham [8]. 


$$
\begin{aligned}
& <\pi^{+}(p)\left|S_{\mu}^{\nu(+)}\right| \bar{K}^{0}(q)>=\frac{1}{(2 \pi)^{3}} \frac{e^{i(p-q) x}}{\sqrt{4 E_{k} E_{\pi}}}, \mathrm{x} \\
& \times\left[(p+q)_{\mu} F_{1}(t)+(q-p)_{\mu} F_{2}(t)\right] . \\
& t=(p-q)^{2}
\end{aligned}
$$

We remark that as a result of the incomplete conservation of $S_{\mu}^{\nu(+)}$ we have two form factors. This means that a complete description of physical processes, like $K \rightarrow \pi e v$, requires a knowledge of both of them. In addition, due to the large $K, \pi$ mass difference, the variation of the two form factors in the $K$ decay spectrum, $0 \leqslant t \leqslant\left(m_{K}-m_{\pi}\right)^{2}$, can be appreciable. Our considerations will be based on the use of the operator $S^{(+)^{K}}$, the "weak chargen derived from $S_{\mu}^{v(+)}$, which is defined as

$$
\begin{aligned}
& S^{(+)}=\int S_{0}^{\nu(+)} d \stackrel{\oplus}{x}=G_{0 s} K_{+} \\
& S^{(-)}=S^{(+)^{*}}
\end{aligned}
$$

(all operators are taken at zero time).

If we form the matrix element $\left\langle\pi\left|S^{(+)}\right| K\right\rangle$, the three-dimensional integration implied by (4.3) gives momentum conservation and we get

$$
<\pi^{+}(p)\left|S^{(+)}\right| \bar{K}_{0}(q)>=\delta(\vec{p}-\vec{q}) G(p)
$$

where

$$
\begin{aligned}
& G(p)=\frac{1}{\sqrt{4 E_{k} E_{\pi}}} \times\left[\left(E_{k}+E_{\pi}\right) F_{1}(t)+\left(E_{k}-E_{\pi}\right) F_{2}(t)\right] \\
& t=\left(E_{k}-E_{\pi}\right)^{2}=\left(\sqrt{\bar{p}^{2}+m_{k}^{2}}-\sqrt{\bar{p}^{2}+m^{2}}\right)^{2}
\end{aligned}
$$

Due to the large mass difference, the matrix element of the charge is not a constant but it depends on the common momentum " $p "$. However, it is important to remark that the momentum transfer $t$ runs in the interval $0 \leqslant t \leqslant\left(m_{K}-m_{\pi}\right)^{2}$, which just corresponds to the physical region of the $K \rightarrow \pi e v$ decas (assuming $\left.m_{e} \sim 0\right)$, so that in the limit of SU(3) validity $\left(m_{K} \rightarrow m_{\pi}\right)$ all $G(p)$ coincide. In particular we note the values of $G(p)$ at the end points of the spectrum:

$$
\begin{aligned}
& G(\infty)=F_{1}(0) \\
& G(0)=\frac{1}{\sqrt{4 m_{k} m_{\pi}}} \times\left[\left(m_{k}+m_{\pi}\right) F_{1}\left(t_{0}\right)+\left(m_{k}-m_{\pi}\right) F_{2}\left(t_{0}\right)\right] \\
& t_{0}=\left(m_{k}-m_{\pi}\right)^{2}
\end{aligned}
$$


The fact that $G$ depends on $p$ makes it clear that using the commutation relations of the $S$, we will derive a continuous set of sum rules for $G(p)$, corresponding to the different values of $p$. $A$ priori they are all on the same footing and indeed they will have some common properties. However, in the following we will give explicit arguments which will make possible a choice of the value of $t$ at which the sum rule is the most favourable in order to study the renormalization effects. Thus as in the previous section we start from the general commutation relation

$$
\left[S^{(+)}, S^{(-)}\right]_{-}=G_{0 s}^{2}\left[K_{+}, K_{-}\right]=G_{0 s}^{2}\left(I_{3}+\frac{3}{2} y^{\prime}\right)=G_{0 s}^{2}(?+Y)
$$

where $Q$ is the total charge, in " $e^{n}$ units, and $Y$ is the hypercharge.

Then we take the expectation value of (4.7) between physical $\pi^{+}$states, introduce a complete set of intermediate states and select the contribution of the $\bar{K}_{0}$ :

$$
G_{0 s}^{2} \delta\left(\vec{p}_{2}-\vec{p}_{1}\right)=G^{2}(p) \delta\left(\vec{p}_{2}-\vec{p}_{1}\right)+\delta G^{2}(p) \delta\left(\vec{p}_{2}-\vec{p}_{1}\right)
$$

where

$$
\begin{aligned}
& \delta G^{2}(p) \delta\left(\vec{p}_{2}-\vec{p}_{1}\right)=\sum_{\alpha \neq \bar{k}_{0}}<\pi^{+}\left|S^{(+)}\right| \alpha> \\
& <\alpha\left|S^{(-)}\right| \pi^{+}>-\sum_{\beta}<\pi^{+}\left|S^{(-)}\right| \rho><\beta\left|S^{(+)}\right| \pi^{+}>
\end{aligned}
$$

Now, following the lines of our previous reasoning, we take into account the existence of a part of the Hamiltonian $H_{1}$, of strength $f$, which breaks $S U(3)$ symmetry and such that the mass splittings are of the first order in $f$. Then we use the relations

$$
\left\langle a\left|S^{( \pm)}\right| b\right\rangle=f \frac{\left\langle a\left|\left[S^{( \pm)}, H_{1}\right]-\right| b\right\rangle}{E_{b}-E_{a}}= \pm f G_{0 s} \frac{\left\langle a \mid I_{s}^{( \pm)} b\right\rangle}{E_{b}-E_{a}}
$$

where $G_{0 s} H_{s}^{( \pm)}=\left[S^{( \pm)}, H_{1}\right]_{-}$can be also expressed in terms of the scalar quantity $f D_{s}^{( \pm)}=$ $\left.\left(\partial K_{\mu}{ }^{( \pm)}\right) / \partial x_{\mu}\right)$, i.e.

$$
H_{s}^{( \pm)}= \pm i \int D_{s}^{( \pm)} d \vec{x}
$$

Finally

$$
\begin{aligned}
& \delta G^{2}(p)=f^{2} \sum_{\alpha \neq \bar{k}_{0}} G_{0 s}^{2} \frac{<\pi^{+}\left|D_{s}^{(+)}\right| \alpha><\alpha\left|D_{s}^{(-)}\right| \pi^{+}>}{\left(E_{\pi}-E_{\alpha}\right)^{2}} \delta\left(\vec{P}_{\alpha}-\vec{p}\right) \\
& -f^{2} \sum_{\beta} G_{0 s}^{2} \frac{<\pi^{+}\left|D_{s}^{(-)}\right| \beta><\beta\left|D_{s}^{(+)}\right| \pi^{+}>}{\left(E_{\pi}-E_{\beta}\right)^{2}} \delta\left(\vec{P}_{\beta}-\vec{p}\right)
\end{aligned}
$$

Let us now discuss in detail the various features of equation (4.12), which represents the central result of this section. First of all, as in the case of electromagnetic corrections, 
we notice the presence of the damping factor $1 /\left(E_{\pi}-E_{\alpha}\right)^{2}$. Secondly, we see that the deviation of $G_{0 s} / G(p)$ from unity is of the order $f^{2}$, i.e., of the order of the deviations from the mass formula.*

This reproduces an important result by Ademollo and Gatto [11], obtained in a different way starting from different hypotheses.** We note that our result does not depend on the particular kind of group used but only on the cancellations connected with quasi-conserved currents.

A third point is represented by the fact, already anticipated, that we have to deal with a continuous set of sum rules corresponding to the different values of $p$. We can verify directly that all $G(p)$ (as shown explicitly by equation (4.5)) differ in order $f^{2}$. Indeed we have

$$
\begin{aligned}
& 0 \leqslant t \leqslant\left(m_{k}-m_{\pi}\right)^{2} \sim f^{2} \\
& \frac{m_{k}-m_{\pi}}{\sqrt{4 m_{k} m_{\pi}}}-1 \sim f^{2} \\
& \frac{m_{k}+m_{\pi}}{\sqrt{4 m_{k} m_{\pi}}}-1 \sim f^{2}
\end{aligned}
$$

and finally, as it will be shown in the next section,

$$
F_{2} \sim f
$$

Those estimates show that all equation (4.8) are consistent among themselves [10].

It is important now to make a comparison between the different sum rules (4.8). Clearly the total quantity $G^{2}(p)+\delta G^{2}(p)$ is independent of $p$ but different choices for $p$ can lead to different relative values of $G^{2}$ and $\delta G^{2}$, i.e., to a different splitting between the zero order term and the corrections. On the other hand, in the limit of validity of $S U(3)$, all $G(p)$ coincide and we may ask which of them has really to be identified with $G_{0}$. The answer to this question is relevant because it would enable us to identify, without ambiguities, which are the best kinematical factors that must be included when we apply $S U(3)$ invariance to physical matrix elements. It goes without saying that this problem, of academic interest in the isospin case of the previous section, is quite important here owing to the large mass differences. ${ }^{+}$

We want to show now that this answer can indeed be given in the framework of our method. In our language this corresponds to giving a criterion of choice between the different sum rules.

- One amusing point is to remark that the $f^{2}$ terms come from the use of equation (4.10) between $\pi$ and higher states than the $K$ meson. Using the same relation between $\pi^{+}$and $\bar{K}_{0}$ states one gets

$$
<\pi|s| \bar{k}>\sim f G_{0 s}<\pi|D| \cdot \bar{k}>/ E_{k}-E_{\pi} \times \delta(\vec{\pi}-\vec{k})
$$

which is of order one because $E_{K}-E_{\pi}$ is of order $f$.

* We thank Dr. G. Segre for an interesting discussion on this point.

+ One could have turned the argument around and used the comparison between the different $G(p)$ to prove that $F_{2}$ is of order $f$. 
It is reasonable to define as the "best" sum rule the one in which the correction $\delta \zeta_{1}^{2}$ is as small as possible. We shall see that a comparison between the different sum rules can be done through very simple kinematical considerations, without any explicit evaluation of the corrections. * To simplify the discussion we consider the case where the intermediate state is given by a particle (or a resonance) of mass $M_{\alpha}$. This does not imply a loss of generality because more complicated cases can be treated in an analogous way.

The general structure of the correction is

$$
\begin{aligned}
& \delta G^{2}(p) \simeq f^{2} G_{0}^{2} \frac{<\pi(p)\left|D^{(+)}\right| \alpha><\alpha\left|D^{(-)}\right| \pi(p)>}{\left(E_{\pi}-E_{\alpha}\right)^{2}} \delta\left(\vec{p}-\vec{p}_{\alpha}\right) \\
& E_{\alpha}=\sqrt{\bar{p}^{2}+M_{\alpha}^{2}}, \quad E_{\pi}=\sqrt{\bar{p}^{2}+m_{\pi}^{2}}
\end{aligned}
$$

Remembering that $I^{ \pm}$are true scalars:

$$
\begin{aligned}
& <\pi(p)\left|D^{(+)}\right|_{\alpha}>=\frac{d_{\alpha}(\bar{t})}{\sqrt{4 E_{\pi} E_{\alpha}}} \\
& \bar{t}=\left(E_{\pi}-E_{\alpha}\right)^{2}=\left(\sqrt{\bar{p}^{2}+m_{\pi}^{2}}-\sqrt{\overline{p^{2}+M_{\alpha}^{2}}}\right)^{2}
\end{aligned}
$$

Equation (4.14) can be rewritten as follows

$$
\delta G^{2}(p) \simeq f^{2} G_{0}^{2} \frac{\left|d_{\alpha}(\bar{t})\right|^{2}}{\left(m_{\pi}^{2}-M_{\alpha}^{2}\right)^{2}} \frac{\left(E_{\pi}+E_{\alpha}\right)^{2}}{\left(\sqrt{\left.4 E_{\pi} E_{\alpha}\right)^{2}}\right.}
$$

Thus the difference between the various, possible sum rules depends on the factor

$$
\varphi(P)=\left|d_{\alpha}(\bar{t})\right|^{2} \frac{\left(E_{\pi}+E_{\alpha}\right)^{2}}{\left(\sqrt{\left.4 E_{\pi} E_{\alpha}\right)^{2}}\right.}
$$

The kinematical factor $\left(E_{\pi}+E_{\alpha}\right)^{2} /\left(\sqrt{4 E_{\pi} E_{\alpha}}\right)$ varies between the maximum value $\left(m_{\pi}+M_{\alpha}\right)^{2} /$ $\left(\sqrt{4 m_{\pi} M_{\alpha}}\right)$, at $p=0$ and the minimum value 1 as $p \rightarrow \infty$. As far as the effect of the form factor $\left|d_{\alpha}(\bar{t})\right|^{2}$ is concerned we notice that $\bar{t}$ ranges between $\left(M_{\alpha}-m_{\pi}\right)^{2}$, for $p=0$, and 0 for $p \rightarrow \infty$. Now it is physically reasonable to assume that the form factor is an increasing function of $\bar{t}$ when $\bar{t}$ becomes larger and time-like (i.e., when $\bar{t}$ approaches and subsequently enters the region of singularities. Thus $d_{\alpha}(\bar{t})$ will be the smallest possible at $\bar{t}=0$, i.e., $p \rightarrow \infty$.

* This evaluation will be discussed in a separate paper. 
Thus on the basis of this model we are led to the conclusion that the quantity $\phi(p)$ is a minimum when $p \rightarrow \infty$, and according to our previous discussion this fixes the "best" sum rule, i.e., that one which makes $G^{2}$ closest to its $S U(3)$ limit $G_{0}^{2}$. Accordingly, it is quite natural to define the renormalized coupling constant (for the $K \pi$ part) as $G_{K \pi}=G(\infty)=F_{1}(0)$, as is usually done. We get for $G$ the relation

$$
G_{0}^{2}=G_{k \pi}^{2}+\delta G^{2}(\infty)
$$

or for the renormalization ratio

$$
\frac{G_{0}^{2}}{G_{k \pi}^{2}}=\frac{Z_{1}^{2}(k \pi)}{Z_{2}(K) Z_{2}(\pi)}=1+\frac{\delta G^{2}(\infty)}{G_{k \pi}^{2}}
$$

\section{Generalized Sum Rules}

In the previous sections we have concentrated our attention on the universality problem, and we were able to get sum rules for the renormalization ratio or, more precisely, for the combination $G(p)$ of equation (4.5). In those formulae the corrections to universality were of order $f^{2}$ and we recognized the reason for this in the presence of the square of the nondiagonal matrix element of $S$, which is of order $f$. In other words the problem of looking for relations between bare and dressed coupling constants leads necessarily to the consideration of commutators of the $Q$ and $S$ with themselves (which are the commutation relations which define the algebra of the group). On the other hand, since the $Q$ and $S$ are generators of a group we know the commutation relations between them and any operator which has definite properties of transformation with respect to the same group. In this way it is possible to pass, after commutation with the suitable $Q$, from one to another member of the multiplet and then to compare different physical processes, by considering the corresponding matrix elements.

The range of application of this technique is quite broad, and in this section we want to illustrate the procedure with some simple examples.

We consider again the case of the $\Delta S=\Delta Q$ vector current. Up to now we have studied only the quantity $G(p)$ and we have obtained the "best" definition of universality. It is interesting to look at the analogous problem for the form factors $F_{1}$ and $F_{2}$. In other words we want to discuss what is the best way to determine $F_{1}$ and $F_{2}$ and their $S U(3)$ limit.

To this end we can use the following commutation relation

$$
\left[S_{\mu}^{(+)}(\bar{x}), S^{(-)}\right]_{-}=G_{0 s}^{2}\left(J_{\mu}^{(0)}(\bar{x})+Y_{\mu}(\bar{x})\right)
$$

where $J_{\mu}{ }^{(0)}$ is the third component of the $\Delta S=0$ current, i.e., the isovector part of the electric current in inits of $e$, and $Y_{\mu}$ is the hypercharge current.

We take the expectation value between physical $\pi^{+}$states and on invariance grounds $\left(J_{\mu}(0)\right.$ is conserved) we get*

- $\left\langle\pi^{+}\left|Y_{\mu}\right| \pi^{+}\right\rangle$is zero as can be seen, for instance, using $G$ parity arguments. 


$$
\begin{aligned}
& <\pi_{1}^{+}\left|\left[S_{\mu}^{(+)}, S^{(-)}\right]_{-}\right| \pi_{2}^{+}>=G_{0 s}^{2}<\pi_{2}^{+}\left|J_{\mu}^{(0)}\right| \pi_{1}^{+}>= \\
& =\frac{G_{0 s}^{2}}{(2 \pi)^{3}} \frac{\left(p_{1}+p_{2}\right) \mu}{\sqrt{\overline{4 E_{1} E_{2}}}} F_{\pi}(t) \\
& t=\left(p_{1}-p_{2}\right)^{2}
\end{aligned}
$$

where $F_{\pi}(t)$ is the pionic electromagnetic form factor. Insertion of intermediate states gives

$$
\begin{gathered}
<\pi_{1}^{+}\left|S_{\mu}^{(+)}\right| \vec{k}_{0}><\bar{k}_{0}\left|S^{(-)}\right| \pi_{2}^{+}>+\delta S_{\mu}\left(p_{1}, p_{2}\right)= \\
=\frac{G_{0 s}^{2}}{(2 \pi)^{3}} \frac{\left(p_{1}+p_{2}\right)_{\mu}}{\sqrt{4 E_{1} E_{2}}} F_{\pi}(t)
\end{gathered}
$$

where as usual

$$
\begin{aligned}
\delta S_{\mu}\left(p_{1}, p_{2}\right) & =f \sum_{\alpha \neq k_{0}} G_{0 s} \frac{<\pi_{1}^{+}\left|S_{\mu}^{(+)}\right| \alpha>\alpha\left|H_{s}^{(-)}\right| \pi_{2}^{+}>+}{E_{\alpha}-E_{2}} \\
& +f \sum_{\beta} G_{0 s} \frac{<\pi_{1}^{+}\left|H_{s}^{(-)}\right| \beta><\beta\left|S_{\mu}^{(+)}\right| \pi_{2}^{+}>}{E_{\beta}-E_{1}}
\end{aligned}
$$

We notice that the corrections contained in $\delta S_{\mu}\left(p_{1}, p_{2}\right)$ are of the first order in the symmetry breaking interaction. The reason for this is that while the non-diagonal elements of $Q$ are small (in the sense that $Q$ is conserved as $f \rightarrow 0$ ), the same dœs not happen for the current. In addition the sum over the intermediate states involves one denominator only, so that the contribution of the higher states is not as strongly damped as in the previous sum rule.

Using equations (4.2) and (4.5) we have

$$
\begin{gathered}
G\left(p_{1}\right) \times \frac{1}{\sqrt{4 E_{2} E_{k}}} \times\left[\left(p_{2}+q\right)_{\mu} F_{1}(\bar{t})+\left(q-p_{2}\right)_{\mu} F_{2}(\bar{t})\right]+ \\
\quad+(2 \pi)^{3} \delta S_{\mu}=G_{0 s}^{2} \frac{\left(p_{1}+p_{2}\right)_{\mu}}{\sqrt{4 E_{1} E_{2}}} F_{\pi}(t)
\end{gathered}
$$

where $q$ is the momentum of the $\bar{K}$ meson

$$
q \equiv\left(\vec{p}_{1}, \sqrt{\bar{p}_{1}^{2}+m_{k}^{2}}\right)
$$


and

$$
\bar{t}=\left(q-p_{2}\right)^{2}
$$

Now in equation (5.5) we are faced with the same problem as before, namely with the choice of the best sum rule. To find it we have to look for the configuration of $p_{1}$ and $p_{2}$ which minimizes the correction $\delta S_{\mu}$. To this end we take $\left|\vec{p}_{1}\right|$ and $\left|\vec{p}_{2}\right|$ both large but $\vec{p}_{1}-\vec{p}_{2}=\vec{k}$ fixed and it is easily seen, with the same arguments as before, that $\delta S_{\mu}$ reaches its minimum value when $\left|\vec{p}_{1}\right|$, $\left|\bar{p}_{2}\right| \rightarrow \infty$. Moreover in this limit

$$
\begin{aligned}
& \left(q+p_{2}\right)_{\mu} \simeq\left(p_{1}+p_{2}\right)_{\mu} \simeq\left(2 \vec{p}_{1}+\vec{k}, 2 p\right) \\
& \left(q-p_{2}\right)_{\mu} \simeq\left(p_{1}-p_{2}\right)_{\mu} \cong(\vec{k}, 0) \\
& t \simeq \bar{t}=-\bar{k}^{2}
\end{aligned}
$$

so that by comparison equation (5.5) furnishes the relations valid for any $t$

$$
\begin{aligned}
& F_{1}(t)=G_{0 s} F_{\pi}(t)+f \delta F_{1} \\
& F_{2}(t)=f \delta F_{2}
\end{aligned}
$$

where $\delta F_{1}, \delta F_{2}$ are the components of $\delta S_{\mu}$ along $\left(p_{1}+p_{2}\right)_{\mu}$ and $\left(p_{1}-p_{2}\right)_{\mu}$, respectively, and we used the fact that $G_{0} / G_{K \pi} \simeq 1+0\left(f^{2}\right)$.

Equation (5.7), derived from the best sum rule, allows one unambiguous determination of what has to be taken as the best $S U(3)$ limit of $\left\langle\pi\left|S_{\mu}\right| K>_{.}^{*}\right.$ Moreover the discussion of the previous section shows that for $t \rightarrow 0, \delta F_{1}$ is of order $f_{\bullet}^{* *}$

It is clear that a completely analogous discussion can be given if we want to evaluate, in the $\Delta S=0$ case, the electromagnetic corrections to the form factors of $\pi^{+} \rightarrow \pi^{0} e^{+} v$ decay, for instance.

Another interesting application is represented by the evaluation of the electromagnetic effects in strong interaction physics. We sketch only the argument for the simple problem of

- It is amusing to see what would be the result if we used equation (5.5) in the limit $\left|\bar{p}_{1}\right| \rightarrow 0$. We get:

$$
\begin{aligned}
& F_{1}(\bar{t})=G_{0 s} F_{\pi}(t) \sqrt{m_{k} / m_{\pi}} \times \frac{m_{k}+m_{\pi}}{2 m_{k}}+f \delta \bar{F}_{1} \\
& F_{2}(\bar{t})=G_{0 s} F_{\pi}(t) \sqrt{m_{k} / m_{\pi}} \times \frac{m_{\pi}-m_{k}}{2 m_{k}}+f \delta \bar{F}_{2}
\end{aligned}
$$

** In this context one clearly understands the nature of the Ademollo and Gatto theorem and its analogy with low energy theorems obtained from gauge invariance [12]. 
evaluating the differences between the coupling constants of charged and neutral pions to nucleons.

We remember that the pion-nucleon charge independent interaction can be written in the form

$$
L_{I} \equiv g\left\{\sqrt{2}\left(g_{5}^{(+)} \varphi^{*}+g_{5}^{(-)} \varphi\right)+g_{5}^{(0)} \varphi_{0}\right\}
$$

where the pseudoscalar quantities are defined as follows:

$$
g_{5}^{(i)}=\bar{\psi} \gamma_{5}{ }^{\top} \psi \text {. }
$$

In addition we consider the vector currents

$$
j_{\mu}^{\nu(i)}=\bar{\psi} \gamma_{\mu} T_{1} \psi
$$

Defining the corresponding "charges", we have the commutation relation

$$
\left[Q^{(+)}, G_{5}^{(-)}\right]_{-}=G_{5}^{(0)}
$$

We take the expectation value between physical proton states and introduce a complete set of intermediate states,

$$
\begin{aligned}
<P_{1}\left|G_{5}^{(0)}\right| P_{2}>= & <P_{1}\left|Q^{(+)}\right| N><\left|G_{5}^{(-)}\right| P_{2}>+ \\
& +\sum_{\alpha \neq N}<P_{1}\left|Q^{(+)}\right| \alpha>\alpha\left|G_{5}^{(-)}\right| P_{2}>- \\
& -\sum_{\beta}<P_{1}\left|G_{5}^{(-)}\right| \beta><\beta\left|Q^{(+)}\right| P_{2}>
\end{aligned}
$$

If we define the following coupling constants*

$$
\begin{aligned}
& <P_{1}\left|G_{5}^{(0)}\right| P_{2}>=G_{p p \pi_{0}} \delta\left(\vec{p}_{2}-\vec{p}_{1}\right) \\
& <P_{1}\left|G_{5}^{(-)}\right| N_{2}>=G_{P N \pi^{+}} \delta\left(\vec{p}_{2}-\vec{p}_{1}\right)
\end{aligned}
$$

Equation (5.12) becomes, using the smallness of the non-diagonal matrix element of $Q^{(+)}$,

- Actually the conventional definition $g_{N N^{\prime} \pi}$ of the coupling constant corresponds to taking

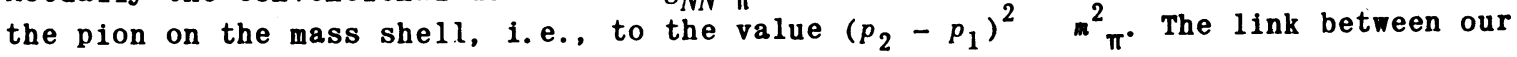
quantities and the conventional ones is

$$
G_{N N^{\prime} \pi}=g_{N N^{\prime} \pi} F_{N N^{\prime} \pi}
$$

where $F_{N N^{\prime} \pi}$ is the $\pi N$ form factor. 


$$
\begin{gathered}
G_{P P_{\pi} 0} \delta\left(\vec{p}_{2}-\vec{p}_{1}\right)=G_{P N \pi}+\delta\left(\vec{p}_{2}-\vec{p}_{1}\right)+ \\
+e \sum_{\alpha \neq N} \frac{<P_{1}\left|H^{(+)}\right| \alpha>}{E_{\alpha}-E_{P}} \times \alpha\left|G_{5}^{(-)}\right| P_{2}>+ \\
+e \sum_{\beta}<P_{1} \mid G_{5}^{(-)|\beta>\beta \beta| H^{(+)} \mid P_{2}>}
\end{gathered}
$$

It is easy to see now that the correction is of order $e^{2}$. In fact, in this order a first contribution is given by states containing one photon and this part introduces amplitudes of the photoproduction type. A second contribution comes from the exchange of a virtual photon between the $P$ and the $\alpha$ states, with the presence of a Compton-like amplitude $\left\langle P\left|j^{(+)} D_{c} j^{(0)}\right|_{\alpha}\right\rangle$.

\section{Acknowledgements}

We want to thank Professors L. Van Hove, Drs. R. Rossetti and G. Segre for very useful discussions.

\section{References}

1. M. GELL-MANN, Phys. Rev. 125, 1067 (1962).

2. G. KaLLEN, Helv. Phys. Acta 25, 417 (1952);

H. LaHMANA, Nuovo Cimento 11, 342 (1954).

3. W. CINI and S. FUBINI, Nuovo Cimento 3, 764 (1956).

4. T. KUMMER, H. PIETSCHMANN and A. BALACHANDRAN, Annals of Physics 29, 161 (1964).

5. S. охово, Nuovo Cimento 13, 292 (1959).

6. R.P. FEYNMAN and M. GBLL-MANN, Phys. Rev. 109, 193 (1958).

7. S. DRELL and J.D. MALECKA, Annals of Physics 28, 18 (1964).

8. M. CINI, E. FERRARI and R. GATTO, Phys. Rev. Letters 2, 7 (1959).

W.N. COTTINGHAM, Annals of Physics 25, 424 (1963).

9. M. GELL-MANN, California Institute of Technology Report CTSL-20, 1961 (unpublished);

Y. NE' EMAN, Nucl. Phys. 26, 222 (1961).

10. N. CABIBBO, Phys. Rev. Letters 10, 531 (1963).

11. M. ADENOLLO and R. GATTO, Phys. Rev. Letters 13, 264 (1965);

see also C. BOUCHIAT and Ph. MEYER, CERN preprint 9321 (1964). 
12. T. ThIRring, Phil. Mag. 41, 1193 (1950);

F. LOT, Phys. Rev. 96, 1428 (1954);

M. GELL-MANN and M.L. GOLDBERGER, Phys. Rev. 96, 1433 (1954);

M. KROLL and A. RUDERMAN, Phys. Rev, 93, 233 (1954. 